\title{
VERIFICATION APPROACHES FOR THE 3D STATIC LES SIMULATIONS OF THE STONECUTTERS BRIDGE DECK
}

\author{
ANTONIO J. ÁLVAREZ, FÉLIX NIETO \& SANTIAGO HERNÁNDEZ \\ University of La Coruña, Spain
}

\begin{abstract}
In computational fluid dynamics (CFD) simulations, verification is the process of identifying both the spatial and the temporal discretisations providing relatively insensitive model results. The underlying goal of this task is to identify the mesh with a lower number of elements and the longer time step size compatible with the purposes of the study. In this manner, the computational burden associated with CFD methods may be decreased without compromising the accuracy of the numerical results. In general, this process is accomplished by studying three meshes with increasing discretisation levels (coarse, medium and fine), expecting that the medium one would provide satisfactory results. Once the satisfactory spatial discretisation level is selected, the different time step sizes are studied, taking into account that a Courant number (Co) of 1 is usually considered the maximum allowable value for numerical stability when adopting a LES (Large Eddy Simulation) approach. In this study, a detailed verification study considering two different approaches, providing the uncertainty level of several parameters of interest depending on the spatial and temporal discretisation is reported. The first of them consists in the curve fitting of linear or quadratic curves to selected model outputs; meanwhile the second approach relies on the Richardson extrapolation principle. These two different approaches are applied in the frame of the 3D LES numerical simulations of the Stonecutters bare deck geometry. In the study the focus is put on the sensitivity of the integral parameters, that is the force coefficients and Strouhal number, with the spatial and temporal discretisations.
\end{abstract}

Keywords: LES, stonecutters bridge, verification, uncertainty, force coefficients, Strouhal number.

\section{INTRODUCTION}

The terms of verification and validation must be used carefully as they refer to different specific processes, as it was stressed by Roache [1] in the context of computational fluid dynamics (CFD) computations. In this regard, verification consists of "solving the equations right", therefore it is a mathematical process, meanwhile validation refers to "solving the right equations", for which a sound scientific and/or engineering understanding of the phenomenon under study is needed, if the previous statement has to be fulfilled.

In bridge engineering applications it is common practice to carry out verification studies based on the evolution of certain parameters in three meshes with increasing grid refinement, aiming at reaching results not influenced by the level of discretisation. This procedure has been used in Álvarez et al. [2] and Laima et al. [3], although this kind of studies does not provide uncertainty levels of the parameters under study, which according to Oberkampf and Roy [4] is one of the four key factors to bring credibility and accuracy to the presented results, generating information of quality of a physical phenomenon, process or system.

According to American Society of Mechanical Engineers [5], verification is comprised of code verification and solution verification. The former is related to the accuracy in solving the mathematical model incorporated in the code, meanwhile the latter estimates the numerical accuracy of a particular calculation. Code verification is usually assumed [5], although as reported by Roache [1], it could be done by applying the method of manufactured solutions. Regarding solution verification, it is comprised by round off errors, due to the precision of computers, iterative errors, especially in time dependent solutions, 
and discretisation errors, due to the approximations made to transform the partial differential equations defining the flow into a system of algebraic equations [6]. Discretisation errors decrease as the grid resolution increases and, in general, it is the dominant source of uncertainty in practical CFD applications [6]. In this piece of research it is assumed that both the round-off and iterative errors are negligible, which in any case have to be two to three orders of magnitude lower than the discretisation error in order to guarantee a negligible influence [5].

The methods used in this paper, which to the author's knowledge is the first application of this methods to a bridge engineering application, are based on power series expansions, specifically the one depicted in Celik et al. [7] and the one presented by Eça and Hoekstra [6]. Therefore a quantifiable method is proposed for selecting the mesh with reasonable computational burdens and level of uncertainty.

\section{FORMULATION}

\subsection{Governing equations}

The movement of a fluid is defined by the Navier-Stokes equations. When they are modelled using a Large Eddy Simulation (LES) approach, the original equations are spatially filtered resulting in the following pair of equations [8]:

$$
\begin{gathered}
\frac{\partial \bar{u}_{\imath}}{\partial x_{i}}=0 \\
\frac{\partial \bar{u}_{\imath}}{\partial t}+\frac{\partial \overline{u_{\imath} u_{j}}}{\partial x_{j}}=-\frac{1}{\rho} \frac{\partial \bar{p}}{\partial x_{i}}+\frac{\partial}{\partial x_{j}}\left[v\left(\frac{\partial \bar{u}_{\imath}}{\partial x_{j}}+\frac{\partial \bar{u}_{j}}{\partial x_{i}}\right)+\tau_{i j}^{S}\right]
\end{gathered}
$$

where $\bar{u}$ is the filtered velocity, $\bar{p}$ is the filtered pressure, $x$ is the space coordinate, $t$ is the time, $v$ is the kinematic viscosity and $\rho$ is the fluid density.

Using the Boussinesq assumption, the sub-grid stress tensor is expressed as:

$$
\tau_{i j}^{S}=v_{t}\left(\frac{\partial \bar{u}_{l}}{\partial x_{j}}+\frac{\partial \bar{u}_{j}}{\partial x_{i}}\right)
$$

where $v_{t}$ is the subgrid-scale turbulent viscosity.

The turbulence model selected for the present work is the Smagorinsky model [9]. This models is based on the assumption of equilibrium between the small resolved scales, dissipating the small ones all the energy extracted from the resolved ones. Moreover, it obeys the following equation:

$$
v_{t}=\left(C_{S} \Delta\right)^{2}\left(2 \overline{S_{l \jmath}} \overline{S_{l \jmath}}\right)^{1 / 2}
$$

where $C_{s}$ is the Smagorinsky constant, $\overline{S_{l j}}$ is the filtered strain rate tensor and $\Delta$ is the characteristic spatial length of the filter, related to the mesh size, and defined as the cubic root of the mesh cell volume $\Delta=\left(\Delta V_{i}\right)^{1 / 3}$. 


\subsection{Force coefficients and Strouhal number}

The time dependent force coefficients $\left(\operatorname{drag}\left(C_{d}\right)\right.$, lift $\left(C_{l}\right)$ and moment $\left.\left(C_{m}\right)\right)$ along with the Strouhal $(S t)$ number, also referred to as integral parameters, are calculated according to eqn (5):

$$
C_{d}=\frac{F_{D}}{\frac{1}{2} \rho U^{2} C}, C_{l}=\frac{F_{L}}{\frac{1}{2} \rho U^{2} C}, C_{m}=\frac{M}{\frac{1}{2} \rho U^{2} C^{2}}, S t=\frac{f D}{U}
$$

where $C$ stands for the width of a single box (see Fig. 1), $D$ is the depth of a single box, $\rho$ is the air density, $U$ is the free-stream velocity, $f$ is the dominant frequency of the lift coefficient, and $F_{D}, F_{L}$ and $M$ are the drag and lift forces and moment per unit of length, which were calculated as the spanwise averaging of the integration of the pressure and viscous forces along the twin-box surfaces. The sign convention of the force coefficient is depicted in Fig. 1.

In the following, the time averaged force coefficients values will be referred as $\overline{C_{k}}$ and their standard deviations as $\widetilde{C_{k}}(k=d, l, m)$.

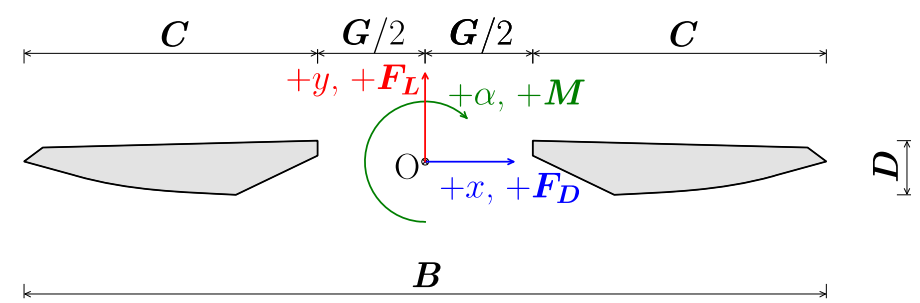

Figure 1: Sign convention.

\subsection{Uncertainty calculation}

For the calculation of the uncertainty due to the spatial discretisation of the integral parameters, the method described in Celik et al. [7], referred to here as "ASME method" and the one described in Eça and Hoekstra [6], named in the following as "Eça's method", are used. As previously commented, both methods are based on power expansion series, whose basic equation for estimation of the discretisation error $(\epsilon)$ is:

$$
\epsilon_{\phi} \simeq \delta_{R E}=\phi_{i}-\phi_{0}=\alpha h_{i}^{p}
$$

where $\phi_{i}$ is the parameter for which the uncertainty is to be calculated, $\phi_{0}$ is the estimated exact solution, $\alpha$ is a constant, $h$ is the representative cell size and $p$ is the observed order of grid convergence.

According to Eça and Hoekstra [6] there are two assumptions that must hold for application of eqn (6): the grids are in the asymptotic range to guarantee that the leading term of the power series expansion is sufficient to estimate the error, and the level of refinement of the mesh can be represented by a single parameter, a representative cell size.

In both methods the representative cell size is calculated as: 


$$
h=\left[\frac{1}{N} \sum_{i=1}^{N}\left(\Delta V_{i}\right)\right]^{1 / 3}
$$

where $N$ is the overall number of elements and $\Delta V$ is the cell volume.

\subsubsection{ASME method}

This method requires of three meshes with increasing refinement level, therefore $h_{1}<h_{2}<h_{3}$, and thus 1 refers to the fine mesh and 3 to the coarse one. The grid refinement factor $r=h_{3} / h_{1}$, has to be greater than 1.3 [7].

Eqns (8)-(11) show how the apparent order $p$ of the method is calculated.

$$
\begin{gathered}
p=\frac{1}{\ln \left(r_{21}\right)}|\ln | \epsilon_{32} / \epsilon_{21}|+q(p)|, \\
q(p)=\ln \left(\frac{r_{21}^{p}-s}{r_{32}^{p}-s}\right), \\
s=1 \cdot \operatorname{sgn}\left(\frac{\epsilon_{32}}{\epsilon_{21}}\right), \\
r_{21}=h_{2} / h_{1}, r_{32}=h_{3} / h_{2}, \epsilon_{21}=\phi_{2}-\phi_{1}, \epsilon_{32}=\phi_{3}-\phi_{2} .
\end{gathered}
$$

Negative values of $\frac{\epsilon_{32}}{\epsilon_{21}}<0$ are indicative of oscillatory convergence. The next step is to calculate the extrapolated values as:

$$
\phi_{\text {ext }}^{21}=\left(l_{21}^{p} \phi_{1}-\phi_{2}\right) /\left(l_{21}^{p}-1\right), \phi_{\text {ext }}^{32}=\left(l_{32}^{p} \phi_{2}-\phi_{3}\right) /\left(l_{32}^{p}-1\right) .
$$

Moreover, along with the apparent order $p$, the approximate relative error $\left(e_{a}\right.$ see eqn (13)), the extrapolated relative error $\left(e_{\text {ext }}\right.$, see eqn (14)) and the grid convergence index for the fine mesh $\left(G C I_{\text {fine }}\right.$, see eqn (15)) has to be calculated and reported.

$$
\begin{gathered}
e_{a}^{21}=\left|\frac{\phi_{1}-\phi_{2}}{\phi_{1}}\right|, \\
e_{\text {ext }}^{21}=\left|\frac{\phi_{\text {ext }}^{12}-\phi_{1}}{\phi_{\text {ext }}^{12}}\right|, \\
G C I_{\text {fine }}^{21}=\frac{1.25 e_{a}^{21}}{r_{21}^{p}-1} .
\end{gathered}
$$

The uncertainty for each grid is calculated as shown in eqn (16). For further information about this method the interested reader is referred to Roache [1] and Celik et al. [7].

$$
U_{1}= \pm G C I_{\text {fine }}^{21} \phi_{1}
$$


2.3.2 Eça's method

In this method three alternatives equations are considered in eqn (6) for estimating the discretisation error:

$$
\begin{gathered}
\epsilon_{\phi} \simeq \delta_{f}=\phi_{i}-\phi_{0}=\alpha h_{i} \\
\epsilon_{\phi} \simeq \delta_{s}=\phi_{i}-\phi_{0}=\alpha h_{i}^{2}, \\
\epsilon_{\phi} \simeq \delta_{f s}=\phi_{i}-\phi_{0}=\alpha_{f} h_{i}+\alpha_{s} h_{i}^{2} .
\end{gathered}
$$

To avoid unreliable results, at least four grids $\left(n_{g} \geq 4\right)$ must be used to obtain a redundant system, which provides a quality check on the value of the apparent order of the method, and the unknowns in eqns (6) and (17)-(19) can be obtained by means of a leastsquares approach. In this regard, the following equations should be minimised:

$$
\begin{aligned}
& S_{R E}\left(\phi_{0}, \alpha, p\right)=\sqrt{\sum_{i=1}^{n_{g}} w_{i}\left(\phi_{i}-\left(\phi_{0}+\alpha h_{i}^{p}\right)\right)^{2}}, \\
& S_{f}\left(\phi_{0}, \alpha\right)=\sqrt{\sum_{i=1}^{n_{g}} w_{i}\left(\phi_{i}-\left(\phi_{0}+\alpha h_{i}\right)\right)^{2}}, \\
& S_{s}\left(\phi_{0}, \alpha\right)=\sqrt{\sum_{i=1}^{n_{g}} w_{i}\left(\phi_{i}-\left(\phi_{0}+\alpha h_{i}^{2}\right)\right)^{2}}, \\
& S_{f s}\left(\phi_{0}, \alpha_{f}, \alpha_{s}\right)=\sqrt{\sum_{i=1}^{n_{g}} w_{i}\left(\phi_{i}-\left(\phi_{0}+\alpha_{f} h_{i}+\alpha_{s} h_{i}^{2}\right)\right)^{2}} .
\end{aligned}
$$

With associated standard deviations:

$$
\begin{gathered}
\sigma_{R E}=\sqrt{\frac{\sum_{i=1}^{n_{g}} n w_{i}\left(\phi_{i}-\left(\phi_{0}+\alpha h_{i}^{p}\right)\right)^{2}}{\left(n_{g}-3\right)}}, \\
\sigma_{f}=\sqrt{\frac{\sum_{i=1}^{n_{g}} n w_{i}\left(\phi_{i}-\left(\phi_{0}+\alpha h_{i}\right)\right)^{2}}{\left(n_{g}-2\right)}},
\end{gathered}
$$




$$
\begin{gathered}
\sigma_{s}=\sqrt{\frac{\sum_{i=1}^{n_{g}} n w_{i}\left(\phi_{i}-\left(\phi_{0}+\alpha h_{i}^{2}\right)\right)^{2}}{\left(n_{g}-2\right)}}, \\
\sigma_{f s}=\sqrt{\frac{\sum_{i=1}^{n_{g}} n w_{i}\left(\phi_{i}-\left(\phi_{0}+\alpha_{f} h_{i}+\alpha_{s} h_{i}^{2}\right)\right)^{2}}{\left(n_{g}-3\right)}} .
\end{gathered}
$$

Non-weighted and weighted approaches are used when performing the calculations. For the non-weighted approach,

$$
w_{i}=1, n=1 \text {, }
$$

meanwhile for the weighted approach,

$$
w_{i}=\frac{1 / h_{i}}{\sum_{i=1}^{n_{g}} 1 / h_{i}}, n=n_{g} .
$$

To calculate the discretisation uncertainty, first, eqn (6) is solved, for both the weighted and non-weighted approach. If the value of $p$ of the two fits is $0.5 \leq p \leq 2$, the apparent order of the method is the one with the smallest standard deviation. If only one of the fits is inside the previous range, $p$ is the one associated with that fit. Otherwise, if the observed apparent order of the method is $p>2$, then eqns (17) and (18) are solved. In case of been $p<0.5$, the eqns (17)-19 have to be solved. In all cases for both approaches, and selecting the apparent order of the method from the fit exhibiting the lower of the standard deviations.

Afterwards, the data range parameter, defined in eqn (30), is calculated in order to assess the quality of the fit.

$$
\Delta_{\phi}=\frac{(\phi)_{\max }-(\phi)_{\min }}{n_{g}-1}
$$

With this value and the apparent order of the method, the safety factor is calculated as:

$$
\left\{\begin{array}{c}
\text { If } 0.5 \leq p<2.1 \text { and } \sigma<\Delta_{\phi}, F_{s}=1.25 \\
\text { Otherwise, } F_{S}=3.0
\end{array} .\right.
$$

Finally the uncertainty is calculated as:

$$
\left\{\begin{array}{c}
\text { If } \sigma<\Delta_{\phi} \Rightarrow U_{\phi}\left(\phi_{i}\right)= \pm\left(F_{s}\left|\epsilon_{\phi}\left(\phi_{i}\right)\right|+\sigma+\left|\phi_{i}-\phi_{f i t}\right|\right) \\
\text { If } \sigma \geq \Delta_{\phi} \Rightarrow \pm U_{\phi}\left(\phi_{i}\right)= \pm 3 \frac{\sigma}{\Delta_{\phi}}\left(\left|\epsilon_{\phi}\left(\phi_{i}\right)\right|+\sigma+\left|\phi_{i}-\phi_{f i t}\right|\right)
\end{array}\right.
$$

For further information on the method the interested reader is referred to Eça and Hoekstra [6] and Rocha et al. [10]. 
Moreover, a slight modification of the previous model is proposed, which consists of setting the safety factor always equal to 1.25 . This modification is based on three points:

- The recommendation of American Society of Mechanical Engineers [5] for using this less conservative value.

- The consideration of acceptable values of $p$ outside of the range described in eqn (31) in Celik et al. [7].

- Eça's method was tested on simulations performed with a modification of the SIMPLE algorithm, which is thought for steady responses, and which is not the case for the application presented in this work.

\section{MODELLING AND COMPUTATIONAL APPROACH}

This study is performed over the geometry of the bare deck cross section of the Stonecutters bridge without modelling the transversal beams linking the boxes. The integral parameters are obtained by means of static 3D LES simulations, which were carried out using the CFD software OpenFOAM. The convective terms were discretised by using the second order upwind differencing scheme, while the second order central difference scheme was applied to the diffusive terms. The second order backward scheme was used for the advancement in time, and finally, the pressure-velocity coupling was solved by the PIMPLE algorithm.

The overall fluid domain is depicted in Fig. 2(a), their main dimensions are shown in Table 1. The spanwise dimension in this study is equal to the width of a single box.

At the inlet Dirichlet conditions were applied to the velocity and turbulent kinetic energy, meanwhile Neumann conditions were imposed to the pressure. At the outlet, Dirichlet conditions were applied to the pressure, and Neumann conditions to the velocity and turbulent kinetic energy. For the upper, lower and lateral faces, symmetric boundary conditions were applied. The incoming flow has a turbulence intensity of $0.0 \%$. In the deck walls no penetration and no-slip boundary conditions were applied.

\section{MESH CHARACTERISTICS}

Two types of meshes were used for the discretisation of the fluid domain, a structured quadrangular one for the boundary layer and in the spanwise dimension, as the meshes were generated by extrusion, and an unstructured quadrangular mesh for the rest of the $X Y$ plane. This plane has been subdivided in different regions, whose outline is depicted in Fig. 2(b).

In Table 2, the non-dimensional sizes of the cells located in the boundaries of each zone are presented. Their size increase as they are placed further away from the deck. For Zone $\mathbf{L}$, only the sizes of the elements located in its right bound are presented, as the size of the elements located in its upper and lower limits grow following a geometric series, starting from the size of Zone $K$, till reaching the one reported for Zone $\mathbf{L}$. The elements of the right borders of Zone $\mathbf{M}$, also increase following a geometric series, from the size of the elements in Zone $\mathbf{L}$ to the ones of Zone $\mathbf{M}$. On the other hand, the characteristics of the boundary layer, the same for all the meshes considered herein, are presented on Table 3.

All the simulations were run at a $R e_{D}=4.48 \cdot 10^{5}$ and Courant number $C o=1$, presenting all the meshes a mean $y^{+}$value, calculated as indicated in Bruno et al. [8], very close to 1 . 


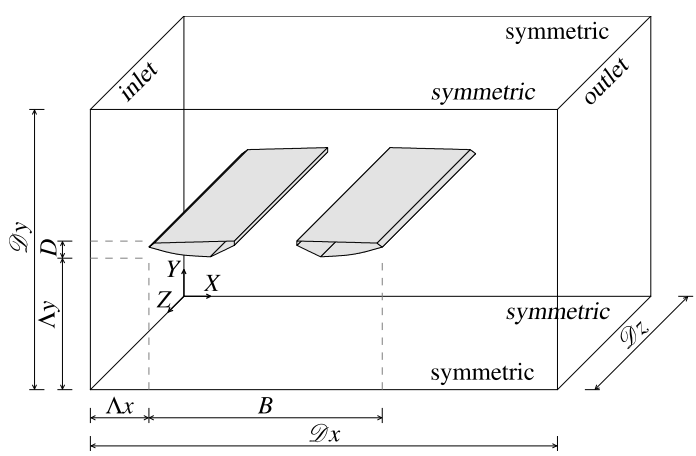

(a)

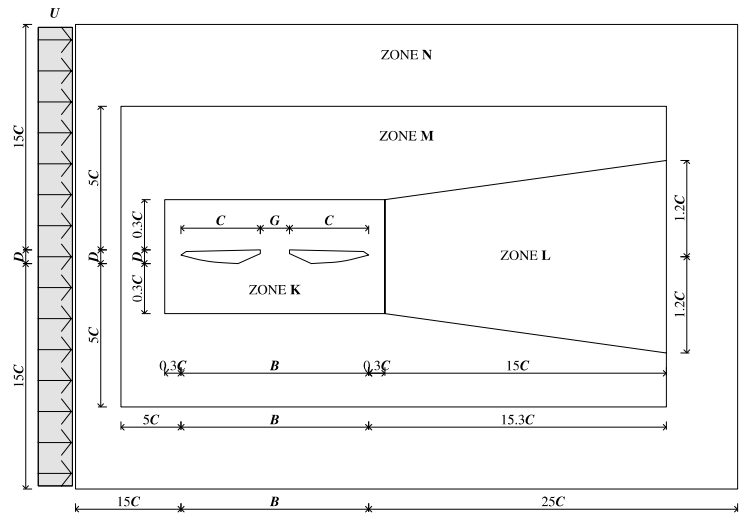

(b)

Figure 2: (a) Overall fluid domain; and (b) Different zones in which the XY plane mesh is subdivided. (Not to scale.)

Table 1: Overall fluid domain dimensions. $\mathrm{B}$ is the deck width, and $\mathrm{C}$ and $\mathrm{D}$ are the width and height of each individual box.

\begin{tabular}{ccccc}
\hline$\Lambda_{x}$ & $\Lambda_{y}$ & $\mathcal{D}_{x}$ & $\mathcal{D}_{y}$ & $\mathcal{D}_{z}$ \\
\hline $15 C$ & $15 C$ & $40 C+B$ & $30 C+D$ & $C$ \\
\hline
\end{tabular}

Table 2: Cell sizes on the borders of the different discretisation zones. The lower the number of the mesh, the higher the level of refinement is. All the values are nondimensional, as the sizes have been divided by $\mathrm{C}$. The computational cost was calculated over a dimensionless time unit $\left(t^{*}=t U / C=1\right)$.

\begin{tabular}{cccccccc}
\hline Mesh & Zone K & Zone L & Zone M & Zone N & \#elements & \#of cores & $\begin{array}{c}\text { Time per } \\
\text { core (hours) }\end{array}$ \\
\hline 1 & 0.008 & 0.083 & 0.208 & 0.418 & 8598192 & 48 & 1.262 \\
2 & 0.014 & 0.109 & 0.251 & 0.559 & 7121232 & 48 & 1.033 \\
3 & 0.021 & 0.145 & 0.33 & 0.8 & 3218112 & 48 & 0.423 \\
4 & 0.031 & 0.2 & 0.45 & 1.0 & 1970208 & 48 & 0.254 \\
\hline
\end{tabular}


Table 3: Boundary layer mesh properties. The parameter $y_{1}$ is the height of the first element of the boundary layer (BL) mesh, $\mathrm{C}$ is the width of a single box, $x_{1}$ is the length of first element in the BL, $r$ is the growth ratio of the elements in the BL, $n_{B L}$ is the number of layers forming the BL mesh, $y_{B L}$ is the total height of the BL mesh, $\delta_{z}$ is the length of the cell in the spanwise dimension and $n_{z}$ is the number of elements in the spanwise dimension.

\begin{tabular}{ccccccc}
\hline$y_{1} / C$ & $x_{1} / y_{1}$ & $r$ & $n_{B L}$ & $y_{B L} / C$ & $\delta_{z} / C$ & $n_{z}$ \\
\hline 0.0007 & 4 & 1.32 & 6 & 0.0088 & 0.021 & 48 \\
\hline \multicolumn{7}{c}{5}
\end{tabular}

5 RESULTS

In Table 4, the values of the integral parameters, obtained from the 3D LES simulations, for all the meshes considered are presented. The convergence criteria followed for the mean and standard deviations of the force coefficients, as well as the Strouhal number, is based on the residuals of the corresponding variables. The residuals for a generic $q$ variable were calculated as $\varphi_{\text {res }}=\left|\left(q_{n}-q_{n-1}\right) / q_{n}\right| \cdot 100$ ( $n$ is the number of sampling windows), and the simulations were extended until the residual value was lower than $5 \%$, as indicated in Bruno et al. [8]. These residuals were obtained for increasing lengths of the sampling window $T_{n}$, with $T_{0}=50$ and $T_{n}=T_{n-1}+50$ (in non dimensional time units $((t U) / D)$ ) (see Fig. 3). These values are the ones used for the calculation of the discretisation uncertainty. In Table 5, the results of applying the ASME method described in Celik et al. [7] are presented, as well as all the mandatory parameters when using this method. They have been calculated using meshes 1,3 and 4 .

Table 4: Integral parameters values for the meshes considered in this study.

\begin{tabular}{cccccccc}
\hline Mesh & $\overline{C_{d}}$ & $\overline{C_{l}}$ & $\overline{C_{m}}$ & $\widetilde{C_{d}}$ & $\widetilde{C_{l}}$ & $\widetilde{C_{m}}$ & $S t$ \\
\hline 1 & 0.151 & -0.263 & 0.226 & 0.023 & 0.166 & 0.076 & 0.247 \\
2 & 0.153 & -0.267 & 0.210 & 0.024 & 0.180 & 0.076 & 0.247 \\
3 & 0.153 & -0.260 & 0.206 & 0.027 & 0.202 & 0.090 & 0.244 \\
4 & 0.154 & -0.244 & 0.183 & 0.033 & 0.244 & 0.118 & 0.235 \\
\hline
\end{tabular}

Continuing with the alternative method (Eça's method), the values of the discretisation uncertainty obtained are presented in Table 6 , in this case the values of the integral parameters provided by the four meshes are used. It can be observed that the uncertainty values yielded by the modified version of the model $\left(U_{i}^{\bmod }, i=1, \ldots, 4\right)$ are smaller than the ones presented by the original version of the method.

It is observed that the ASME method provides higher uncertainty values for coarser meshes, meanwhile this is not always the case for Eça's method. This can be explained by the fact that, in the calculation of the uncertainty shown in eqn (31), one of the elements is the difference between the value of the parameter obtained in the simulation and the fitted one. Moreover, this phenomenon could be explained by the fact of using only four meshes, although further investigation has to be conducted in this regard, and also the demanding computational needs of the 3D LES simulations, makes the increment of the number of simulations a cumbersome task. 


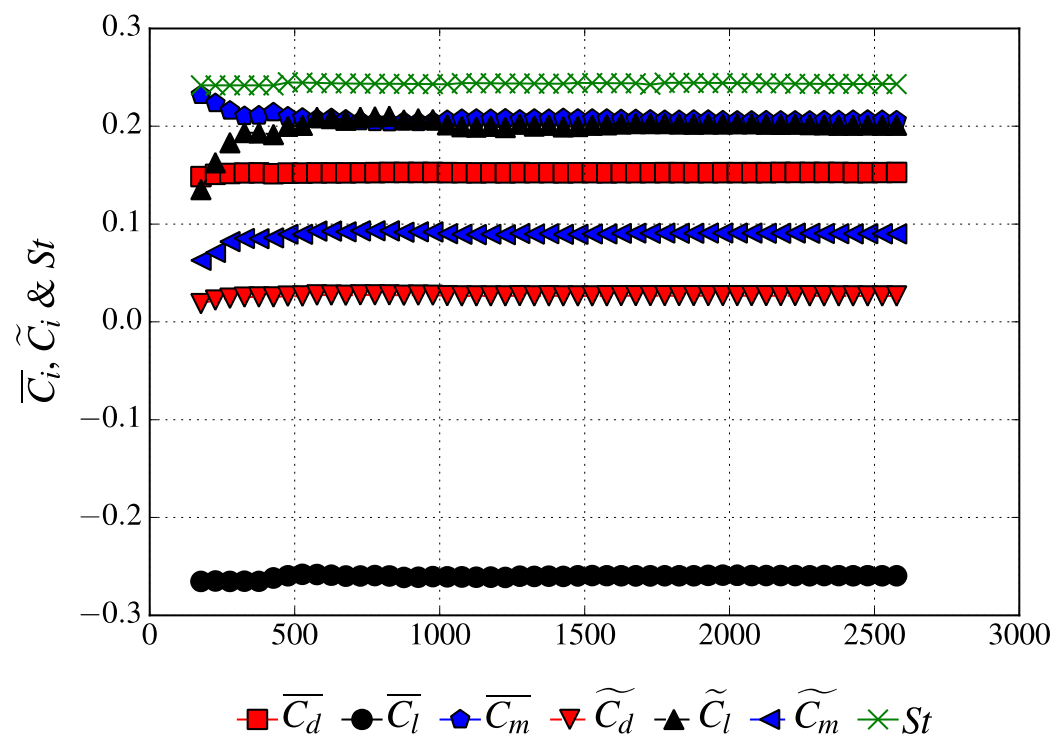

(a)

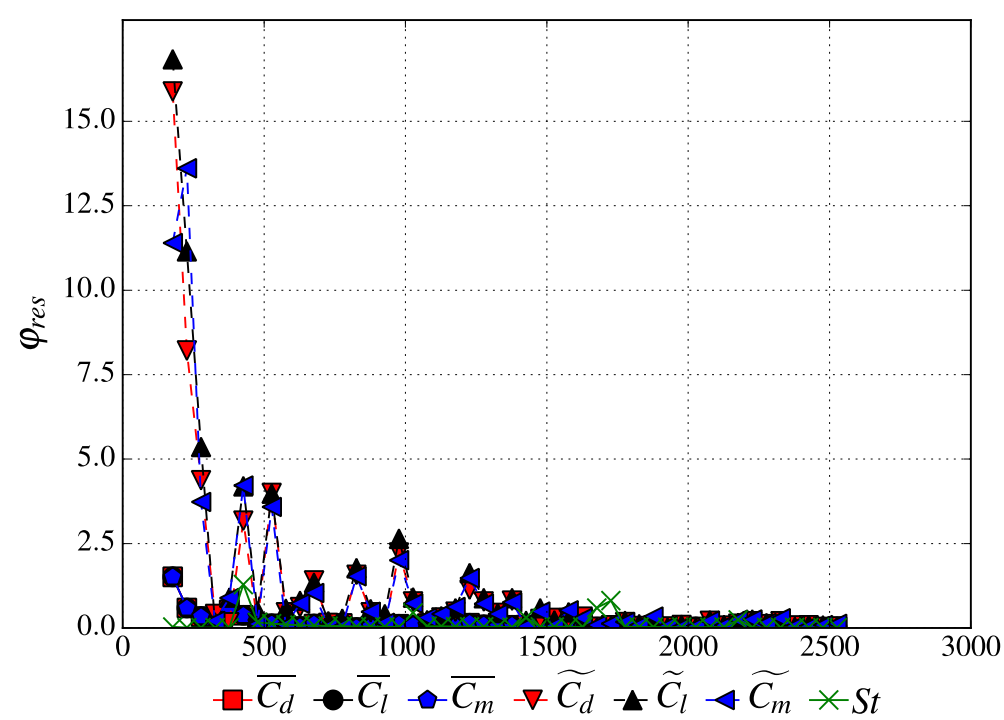

(b)

Figure 3: (a) Integral parameters evolution; and (b) Integral parameters residuals for increasingly longer sampling windows, for mesh 3. Red color refers to statistical properties of the drag coefficient, black for the lift coefficient and blue for the moment coefficient, green color refers to the Strouhal number. 
Table 5: Spatial discretisation uncertainty using the ASME method.

\begin{tabular}{|c|c|c|c|c|c|c|c|}
\hline & $\overline{\overline{C_{d}}}$ & $\overline{\bar{C}_{l}}$ & $\overline{\overline{C_{m}}}$ & $\widetilde{C_{d}}$ & $\widetilde{C}_{l}$ & $\widetilde{C_{m}}$ & $S t$ \\
\hline$N_{1}, N_{3}, N_{4}$ & \multicolumn{7}{|c|}{$8598192,3218112,1970208$} \\
\hline$h_{1}, h_{3}, h_{4}$ & \multicolumn{7}{|c|}{$4.881 e^{-3}, 5.198 e^{-3}, 6.773 e^{-3}$} \\
\hline$r_{31}$ & & & & 1.388 & & & \\
\hline$r_{43}$ & & & & 1.178 & & & \\
\hline$\phi_{1}$ & 0.151 & -0.263 & 0.226 & 0.023 & 0.166 & 0.076 & 0.247 \\
\hline$\phi_{3}$ & 0.153 & -0.260 & 0.206 & 0.027 & 0.202 & 0.090 & 0.244 \\
\hline$\phi_{4}$ & 0.154 & -0.244 & 0.183 & 0.033 & 0.244 & 0.118 & 0.235 \\
\hline$p$ & 2.990 & 11.661 & 3.507 & 4.653 & 3.588 & 6.190 & 7.858 \\
\hline$\phi_{\text {ext }}^{31}$ & 0.150 & -0.263 & 0.236 & 0.021 & 0.149 & 0.074 & 0.248 \\
\hline$\phi_{\text {ext }}^{43}$ & 0.150 & -0.263 & 0.235 & 0.021 & 0.149 & 0.074 & 0.248 \\
\hline$e_{a}^{31}$ & $1.138 \%$ & $1.047 \%$ & $8.999 \%$ & $18.059 \%$ & $21.959 \%$ & $18.417 \%$ & $1.333 \%$ \\
\hline$e_{a}^{43}$ & $1.136 \%$ & $6.202 \%$ & $11.215 \%$ & $22.301 \%$ & $20.791 \%$ & $31.380 \%$ & $3.824 \%$ \\
\hline$e_{\text {ext }}^{31}$ & $0.689 \%$ & $0.023 \%$ & $4.010 \%$ & $5.295 \%$ & $10.874 \%$ & $2.872 \%$ & $0.110 \%$ \\
\hline$e_{\text {ext }}^{43}$ & $1.835 \%$ & $1.070 \%$ & $12.649 \%$ & $24.311 \%$ & $35.221 \%$ & $21.819 \%$ & $1.441 \%$ \\
\hline$G C I_{\text {fine }}^{31}$ & $0.856 \%$ & $0.029 \%$ & $5.222 \%$ & $6.286 \%$ & $12.259 \%$ & $3.492 \%$ & $0.137 \%$ \\
\hline$G C I_{\text {medium }}^{43}$ & $2.253 \%$ & $1.352 \%$ & $18.100 \%$ & $24.446 \%$ & $32.559 \%$ & $22.389 \%$ & $1.828 \%$ \\
\hline$G C I_{\text {coarse }}$ & $9.781 \%$ & $415.186 \%$ & $101.314 \%$ & $240.230 \%$ & $189.642 \%$ & $468.025 \%$ & $86.679 \%$ \\
\hline$U_{\text {fine }}^{31}$ & $1.292 e^{-3}$ & $7.701 e^{-5}$ & $1.180 e^{-2}$ & $1.417 e^{-3}$ & $2.030 e^{-2}$ & $2.658 e^{-3}$ & $3.398 e^{-4}$ \\
\hline$U_{\text {medium }}^{43}$ & $3.441 e^{-3}$ & $3.512 e^{-3}$ & $3.722 e^{-2}$ & $6.506 e^{-3}$ & $6.576 e^{-2}$ & $2.019 e^{-2}$ & $4.459 e^{-3}$ \\
\hline$U_{\text {coarse }}$ & $1.511 e^{-2}$ & 1.012 & $1.849 e^{-1}$ & $7.819 e^{-2}$ & $4.627 e^{-1}$ & $5.546 e^{-1}$ & $2.034 e^{-1}$ \\
\hline $\begin{array}{c}\text { oscillatory } \\
\text { convergence }\end{array}$ & $x$ & $x$ & $x$ & $x$ & $x$ & $x$ & $x$ \\
\hline
\end{tabular}

Table 6: Spatial discretisation uncertainty using Eça's method.

\begin{tabular}{cccccccc}
\hline & $\overline{C_{d}}$ & $\overline{C_{l}}$ & $\overline{C_{m}}$ & $\widetilde{C_{d}}$ & $\widetilde{C_{l}}$ & $\widetilde{C_{m}}$ & $S t$ \\
\hline$\phi_{0}$ & 0.148 & -0.278 & 0.242 & 0.017 & 0.125 & 0.005 & 0.255 \\
$p_{\text {observed }}$ & 1.000 & 4.004 & 3.592 & 4.499 & 3.591 & 4.376 & 4.316 \\
fit & eqn (17) & eqn (18) & eqn (18) & eqn (18) & eqn (18) & eqn (18) & eqn (18) \\
\hline$U_{1}$ & 0.020 & 0.054 & 0.062 & 0.018 & 0.131 & 0.090 & 0.025 \\
$U_{2}$ & 0.029 & 0.039 & 0.114 & 0.021 & 0.176 & 0.087 & 0.027 \\
$U_{3}$ & 0.026 & 0.063 & 0.123 & 0.031 & 0.243 & 0.136 & 0.038 \\
$U_{4}$ & 0.031 & 0.110 & 0.189 & 0.049 & 0.366 & 0.219 & 0.064 \\
\hline$U_{1}^{\text {mod }}$ & 0.020 & 0.027 & 0.033 & 0.008 & 0.060 & 0.042 & 0.012 \\
$U_{2}^{\text {mod }}$ & 0.029 & 0.021 & 0.057 & 0.010 & 0.080 & 0.040 & 0.012 \\
$U_{3}^{\text {mod }}$ & 0.026 & 0.032 & 0.059 & 0.014 & 0.109 & 0.063 & 0.018 \\
$U_{4}^{\text {mod }}$ & 0.031 & 0.050 & 0.085 & 0.021 & 0.159 & 0.096 & 0.029 \\
\hline weighted & $\times$ & $\checkmark$ & $x$ & $\checkmark$ & $\checkmark$ & $\checkmark$ & $\checkmark$ \\
\hline
\end{tabular}

\section{CONCLUSIONS}

In this piece of research the application of two different methods for the calculation of the discretisation uncertainty to the values obtained by means of 3D LES simulations have been presented for a practical case of interest in bridge engineering. The ASME method presented two main advantages respect to Eça's method: (i) it required a lower number of simulations and (ii) it has proved to be less conservative in the calculation of the uncertainty values. This suggests that the ASME method is more suitable for a wider range of practical applications. Moreover, the calculation of the uncertainties of the different parameters of interest provides major overall credibility to the obtained results, offering as 
well a quantifiable method for selecting a mesh with reasonable accuracy and computational demands, which could be used in further studies.

\section{ACKNOWLEDGEMENTS}

This research has been funded by the Spanish Ministry for Science and Innovation in the frame of the research project with reference PID2019-110786GB-I00 and the Galician regional Government with reference ED431C2017/72. The computations have been carried out in the computer cluster Breogan and in the Galician Supercomputing Center (CESGA). The authors fully acknowledge the received support.

\section{REFERENCES}

[1] Roache, P.J., Fundamentals of Verification and Validation, Hermosa: Socorro, New Mexico, 2009.

[2] Álvarez, A.J., Nieto, F., Kwok, K.C.S. \& Hernández, S., A computational study on the aerodynamics of a twin-box bridge with a focus on the spanwise features. Journal of Wind Engineering and Industrial Aerodynamics, 209, pp. 104465-1-18, 2021.

[3] Laima, S., Jiang, C., Li, H., Chen, W. \& Ou, J., A numerical investigation of Reynolds number sensitivity of flow characteristics around a twin-box girder. Journal of Wind Engineering and Industrial Aerodynamics, 172, pp. 298-316, 2018.

[4] Oberkampf, W.F. \& Roy, C.J., Verification and Validation in Scientific Computing, Cambridge University Press: Cambridge, UK, 2010.

[5] American Society of Mechanical Engineers, Standard for Verification and Validation in Computational Fluid Dynamics and Heat Transfer, ASME: New York, 2009.

[6] Eça, L. \& Hoekstra, M., A procedure for the estimation of the numerical uncertainty of CFD calculations based on grid refinement studies. Journal of Computational Physics, 262, pp. 104-130, 2014.

[7] Celik, I.B., Ghia, U., Roache, P.J., Freitas, C.J., Coleman, H. \& Raad, P.E., Procedure for estimation and reporting of uncertainty due to discretization in CFD applications. Journal of Fluids Engineering, 130, pp. 078001-1-4, 2008.

[8] Bruno, L., Fransos, D., Coste, N. \& Bosco, A., 3D flow around a rectangular cylinder: A computational study. Journal of Wind Engineering and Industrial Aerodynamics, 98, pp. 263-276, 2010.

[9] Smagorinsky, J., General circulation experiments with the primitives equations. I: The basic experiments. Month. Weath. Rev., 3(91), pp. 99-165, 1963.

[10] Rocha, A.L., Eça, L. \& Vaz, G., On the numerical convergence properties of the calculation of the flow around the KVLCC2 tanker in unstructured grids. Proceedings of the VII International Conference on Computational Methods in Marine Engineering, pp. 336-352, 2017. 